\title{
Excitonic trions in vertically coupled quantum dots
}

\author{
Egidijus Anisimovas and F. M. Peeters \\ Departement Natuurkunde, Universiteit Antwerpen (UIA), B-2610 Antwerpen, Belgium
}

(Received 26 March 2003; revised manuscript received 27 June 2003; published 16 September 2003)

\begin{abstract}
A theoretical investigation, based on an exact numerical diagonalization, is presented for negatively charged excitonic trions in vertically coupled parabolic quantum dots in the presence of an external magnetic field. The hole and electrons reside in separate dots. We investigate the properties of the system as a function of the interdot separation and an externally applied magnetic field, and observe a number of ground-state angular momentum transitions. At the angular momentum transitions the charge distribution in the dots undergoes a sudden redistribution, leading to jumps in the average electron and hole radii. The interparticle correlations and magnetophotoluminescence oscillator strengths are also discussed.
\end{abstract}

DOI: 10.1103/PhysRevB.68.115310

PACS number(s): 73.21.La, 78.67.Hc

\section{INTRODUCTION}

The quantum-mechanical description of three-particle complexes has a long history dating back to the early days of quantum theory. In atomic physics the paradigm and basic objects of interest were the atomic and molecular hydrogenic ions $\mathrm{H}^{-}$and $\mathrm{H}_{2}{ }^{+}$, while the analogous systems in condensed matter are the negative $\left(\mathrm{X}^{-}\right)$and positive $\left(\mathrm{X}_{2}{ }^{+}\right)$excitonic trions ${ }^{1}$ composed, respectively, of two electrons bound to a hole or vice versa. In bulk crystals, the existence and stability of such and other charged complexes was predicted by Lampert $^{1}$ and confirmed by numerous observations. ${ }^{2-5}$ However, their binding energies (with respect to dissociation to an exciton and a free electron or hole) turned out to be very small, thus making the experimental observation of charged excitons a challenging task.

In artificially structured low-dimensional structures the charge carriers are confined in one or more spatial directions. Thereby the balance of different contributions to the system energy is changed, and as a result, the binding energies of excitonic trions may increase quite substantially. ${ }^{6,7}$ Charged excitons were observed in semiconductor quantum wells ${ }^{8-11}$ and quantum dots. ${ }^{12}$ Also, higher complexes with more than one excess charge can be formed. ${ }^{13-15}$

The increased binding energies in low-dimensional systems is supplemented by a number of additional advantages. Recent progress in measurement techniques allows one to perform photoluminescence measurements on a single quantum dot. ${ }^{15-17}$ In this way, one gets rid of the inhomogeneous broadening and it becomes possible to directly probe the energy states of neutral and charged excitons in a quantum dot. Also, novel approaches based on significantly different diffusion constants of photogenerated electrons and holes make it possible to charge quantum dots by a different number of electrons and holes in a controllable manner. ${ }^{15}$

The excitonic trions in quantum wells were extensively studied by a number of authors. ${ }^{18-20}$ One of the most intriguing issues in quasi-two-dimensional systems in magnetic fields is the fact that the lowest-energy spin-triplet state is optically inactive. ${ }^{21,22}$ The puzzle of the "dark" triplet was solved by discovering a novel exact selection rule ${ }^{22}$ governing the optical transitions of charged excitons which is a manifestation of magnetotranslational symmetry. ${ }^{23}$
Charged excitons in quantum dots were addressed theoretically by Szafran and co-workers. ${ }^{24,25}$ These authors employed a variational trial wave function to study trion binding energies in single and coupled quantum dots in the absence of a magnetic field. Earlier, the interaction of an exciton confined in a quantum dot with an additional electron was studied. ${ }^{26}$

In the present paper, we present a study of negatively charged excitons confined in a two-quantum-dot molecule in the presence of a perpendicular magnetic field. We assume that the electrons and hole are spatially separated. This can be realized by applying a perpendicular electric field to the two-dot layer. Recently it was shown ${ }^{27}$ that the localization of electrons and holes in different dots also occurs due to the very different environment felt by the two kinds of particles. Earlier calculations on trions in quantum well systems ${ }^{20}$ indicated that the trion binding energy was a very sensitive function of the vertical separation between the electrons and hole. Our work will shed new light on this issue in the confined case. We base our approach on exact diagonalizations in the Hilbert space of three-particle configurations. This work is an extension of our earlier investigations of neutral electron-hole complexes in double quantum $\operatorname{dots}^{28}$ (see also Ref. 29) to charged systems.

The structure of the paper is as follows. In Sec. II, we formulate the theoretical approach, present our results in Sec. III, and conclude with the summarizing Sec. IV. The Appendix discusses the calculation of the Coulomb matrix elements.

\section{THEORY}

In our model, the two electrons (mass $m^{*}$, charge $-e$ ) and hole (mass $M^{*}$, charge $+e$ ) move in parallel twodimensional layers separated by a vertical distance $\mathbf{d}$ and are laterally confined by circularly symmetric confining potentials whose strengths we denote by $\omega_{0}$ and $\Omega_{0}$, respectively, for the electrons and hole. Figure 1 shows the geometry of the dots. The magnetic field is included in terms of its symmetric-gauge vector potential $\mathbf{A}=[\mathbf{B} \times \mathbf{r}] / 2$. The complete Hamiltonian reads 


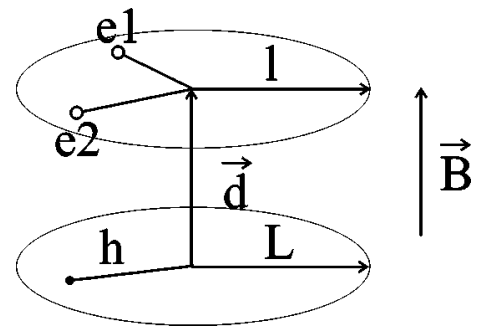

FIG. 1. The geometry of the coupled dots.

$$
H=\sum_{i=1}^{2} T_{e i}+T_{h}+V_{e e}+\sum_{i=1}^{2} V_{e i, h}+H_{Z}
$$

The first two terms denote the single-particle parts, respectively, of the two electrons and hole; the following two terms stand for the electron-electron and electron-hole Coulomb interactions, while the last one is the Zeeman contribution. Both the electrons and hole are treated in the effective mass approximation; thus the single-particle terms are expressed as

$$
\begin{gathered}
T_{e i}=\frac{\hbar^{2}}{2 m^{*}}\left[\mathbf{p}_{e i}+\frac{e}{c} \mathbf{A}\left(\mathbf{r}_{e i}\right)\right]^{2}+\frac{1}{2} m^{*} \omega_{0}^{2} r_{e i}^{2}, \\
T_{h}=\frac{\hbar^{2}}{2 M^{*}}\left[\mathbf{p}_{h}-\frac{e}{c} \mathbf{A}\left(\mathbf{r}_{h}\right)\right]^{2}+\frac{1}{2} M^{*} \Omega_{0}^{2} r_{h}^{2} .
\end{gathered}
$$

The interaction terms are given by

$$
\begin{gathered}
V_{e e}=\frac{e^{2}}{\epsilon} \frac{1}{\left|\mathbf{r}_{e 1}-\mathbf{r}_{e 2}\right|}, \\
V_{e i, h}=-\frac{e^{2}}{\epsilon} \frac{1}{\left|\mathbf{r}_{e i}-\mathbf{r}_{h}+\mathbf{d}\right|} .
\end{gathered}
$$

In the present calculation we include only the electronic spin degree of freedom and assume the hole to be in the spin-up state with $S_{h}^{z}=3 / 2$. This does not compromise the generality since the two distinct subspaces of trion states- the ones defined by the hole being polarized spin up $\left(S_{h}^{z}=3 / 2\right)$ and spin down $\left(S_{h}^{z}=-3 / 2\right)$ - are not connected by the Coulomb interaction. Therefore, the diagonalizations may be carried out separately and produce identical sets of states whose energies differ by $3 g_{h}^{*}$, with $g_{h}^{*}$ being the hole effective gyromagnetic factor. Moreover, these two sets of states will be optically active in the channels of opposite light polarization (see, e.g., the trion energy level diagram in Fig. 4 of Ref. 30). In view of these considerations we will restrict ourselves to the treatment of one of these sets and disregard the hole Zeeman energy. Thus the Zeeman term in Eq. (1) is the Zeeman energy of the electronic subsystem and is given by

$$
H_{Z}=\mu_{B} g_{e}^{*} B S_{e}^{z},
$$

where $S_{e}^{z}$ is the $z$ component of the total electron spin.

We choose to work in dimensionless "atomic" units set by the electron material parameters in GaAs. Thus we use $m^{*}=0.067 m_{e}, \varepsilon=12.4$, and $g_{e}^{*}=-0.44$ and find that the effective Bohr radius (the length unit) becomes $a_{B}^{*}$ $=\hbar^{2} \varepsilon / m^{*} e^{2}=9.8 \mathrm{~nm}$ and the effective Hartree energy (the energy unit) is $E_{H}^{*}=e^{2} / \varepsilon a_{B}^{*}=11.86 \mathrm{meV}$. We take the effective hole mass $M^{*}=0.45 m_{e}$.

As already discussed above, we solve Eq. (1) by performing exact diagonalizations. We will use a basis of many-body configurations which is built of single-electron and singlehole states corresponding to the case when the Coulomb interaction is absent: i.e., the Fock-Darwin states in a magnetic field. For electrons these read

$$
\psi_{e}(n m \mid r \theta)=\frac{\mathrm{e}^{i m \theta}}{\sqrt{\pi}} \sqrt{\frac{n !}{(n+|m|) !}} \mathrm{e}^{-r^{2} / 2 l^{2}} \frac{r^{|m|}}{l^{|m|+1}} L_{n}^{|m|}\left(\frac{r^{2}}{l^{2}}\right) .
$$

Here $l$ is the magnetic-field-renormalized extension of the electron wave function in the dot; it is computed from the zero-field extension $l_{0}=\left(\hbar / m^{*} \omega_{0}\right)^{1 / 2}$ and the magnetic length $l_{c}=(\hbar c / e B)^{1 / 2}$ according to $l^{-4}=l_{0}^{-4}+l_{c}^{-4} / 4$. The expression of the hole states is obtained from Eq. (5) upon replacement of the radius $l \rightarrow L$ with $L^{-4}=L_{0}^{-4}+l_{c}^{-4} / 4$. Note that the condition $l_{0}=L_{0}$ at $B=0$ also implies the equality of wave function sizes at arbitrary magnetic fields since the magnetic length $l_{c}$ does not depend on material parameters. Moreover, even if the zero-field dot sizes are not precisely equal, the presence of a finite magnetic field will reduce their disparity; i.e., the magnetic-field-renormalized dot sizes will differ less. Thus, for the sake of convenience, we assume the two dots to be equal sized. The use of the above-introduced basis is convenient in that the singleparticle part of the Hamiltonian, Eq. (1), is immediately diagonal and the remaining task is the computation of the Coulomb matrix elements and numerical diagonalization of the matrix. The Coulomb matrix elements are calculable analytically to a large extent, and the Appendix gives further details on this matter. We typically include six lowest degenerate shells of single-electron and single-hole states in the calculation and obtain an absolute accuracy on the order of $10^{-3} E_{H}^{*}$. The errors of our method tend to increase several times towards the $d \rightarrow 0$ limit, as the interaction between the hole and electrons increases and thus the basis functions used become less suitable. The total three-particle angular momentum is a good quantum number; therefore, the diagonalizations can be carried out separately in subspaces of different angular momenta, thus considerably reducing the numerical effort.

The single-electron and hole energies in the states (5) are given by

$$
\begin{aligned}
& E_{e}(n, m)=\hbar \sqrt{\omega_{0}^{2}+\frac{\omega_{c}^{2}}{4}}(2 n+|m|+1)+\frac{1}{2} \hbar \omega_{c} m, \\
& E_{h}(n, m)=\hbar \sqrt{\Omega_{0}^{2}+\frac{\Omega_{c}^{2}}{4}}(2 n+|m|+1)-\frac{1}{2} \hbar \Omega_{c} m,
\end{aligned}
$$

with $\omega_{c}=e B / m^{*} c$ and $\Omega_{c}=e B / M^{*} c$ being the electron and hole cyclotron frequencies. Thus, at nonzero magnetic fields 

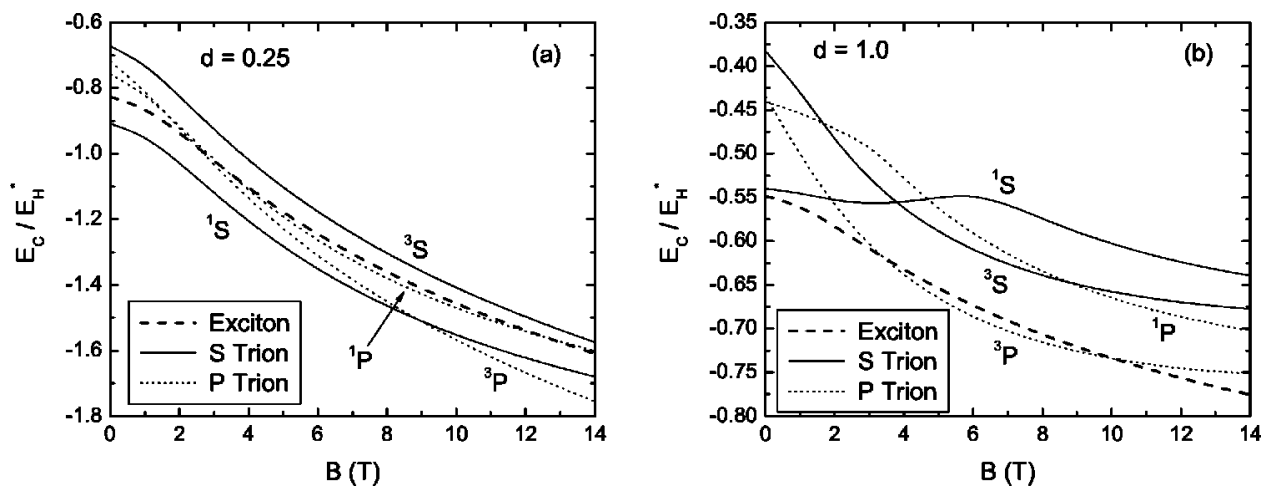

FIG. 2. Correlation energy of a negatively charged trion in coupled quantum dots for two values of interdot distance $d=0.25$ (a) and $d=1.0$ (b). The thick dashed line denotes the correlation energy of an exciton; solid (dotted) lines correspond to $S(P)$ states of a trion. The lowest singlet and triplet states are shown. the electrons will prefer negative angular momenta over positive and vice versa for the holes.

We define the trion (exciton) correlation energy as the difference of the trion (exciton) energy and the ground-state energies of its constituent particles:

$$
\begin{gathered}
E_{C}\left(X^{-}\right)=E\left(X^{-}\right)-2 E(e)-E(h), \\
E_{C}(X)=E(X)-E(e)-E(h) .
\end{gathered}
$$

The trion binding energy is the difference between the energy of an exciton plus a free electron (in a quantum dot) and the trion energy. This quantity can be interpreted as the energy needed to transfer one electron from the quantum-dot molecule confining the trion to another identical empty quantum-dot molecule located at a distance large enough to eliminate Coulomb coupling between the molecules. The binding energy can alternatively be expressed as the difference of the exciton and trion correlation energies:

$$
E_{B}\left(X^{-}\right)=E(X)+E(e)-E\left(X^{-}\right)=E_{C}(X)-E_{C}\left(X^{-}\right) .
$$

Positive (negative) binding energies correspond to bound (unbound) trions.

\section{RESULTS}

\section{A. Energy spectra}

We numerically calculated the energies and states of the electron-hole system defined by Eq. (1). One of the most conspicuous results is the switching of the ground-state angular momentum and spin multiplicity as a function of the vertical separation between the dots $d$ and the magnetic field strength $B$.

In Fig. 2, we display the magnetic field dependence of the trion correlation energies and compare them to the correlation energy of the exciton ground state. We include the lowest singlet and triplet states of angular momenta 0 and -1 -i.e., the ones that are competing for the status of the ground state. As explained previously, the trion states that are found below the exciton line are bound. In Fig. 2(a) the results for two closely spaced dots with $d=0.25$ are shown, and we observe that at intermediate magnetic fields up to three subspaces $\left({ }^{1} S,{ }^{1} P\right.$, and ${ }^{3} P$ ) become bound, while the state ${ }^{3} S$ is never bound. The same behavior persists when the interdot distance is further reduced down to $d=0$. The ground state of the trion switches from ${ }^{1} S$ to ${ }^{3} P$ at a magnetic field value close to $9 \mathrm{~T}$.

The corresponding spectra for more distant dots are shown in Fig. 2(b). Here the interdot separation is set to $d$ $=1.0$ which is close to the trion binding edge (see Fig. 3), and we find only one $\left({ }^{3} P\right)$ state that is weakly bound at intermediate magnetic fields. Quite interestingly, there is a crossing between excited $S$ states, and at $B>3.8 \mathrm{~T}$ the triplet state becomes lower in energy than the singlet state.

In Fig. 3, we depict the $B-d$ phase diagram of the system composed of two equal-sized dots with $l_{0}=L_{0}=2.5 a_{B}^{*}$. The interdot distance is varied between 0 and $5 a_{B}^{*}$-that is, twice the dot radius - and the magnetic field ranges from 0 to $14 \mathrm{~T}$. We use the common spectroscopic notation $(S, P, D, F, \ldots)$ to denote the values of the angular momentum and indicate the spin states by a left superscript 1 and 3 for singlet and triplet, respectively.

The hatched area at low $d$ values denotes the domain where the particles form a bound trion; i.e., its ground state is stable with respect to dissociation into an exciton and an

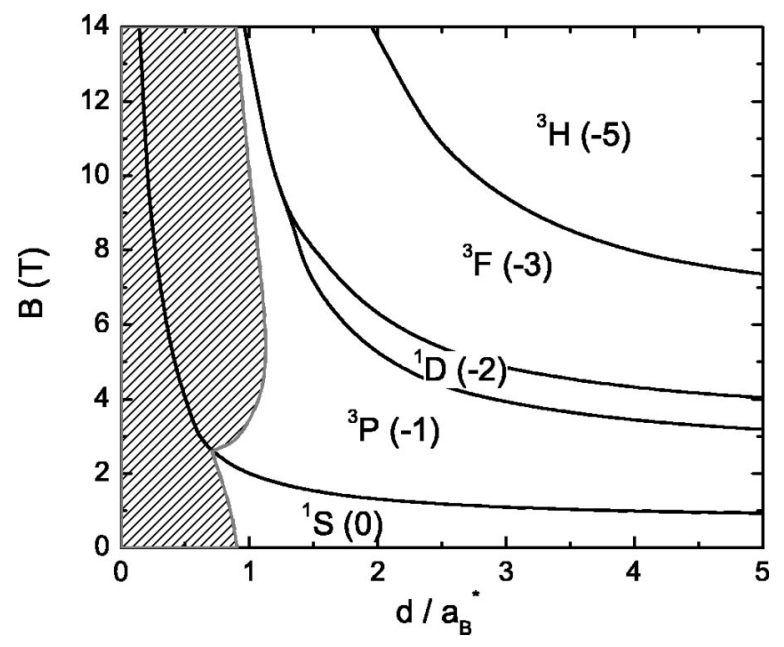

FIG. 3. The phase diagram of a trion confined in a quantum-dot molecule of confinement radii $l_{0}=L_{0}=2.5 a_{B}^{*}$. The thick lines separate areas of different ground-state angular momenta and spin multiplicities. The angular momenta are denoted by a capital letter and its numerical value is also shown in the parentheses. The superscripts 1 and 3 stand for, respectively, singlet and triplet states. The hatched area indicates the range of parameter values at which trions are bound. 

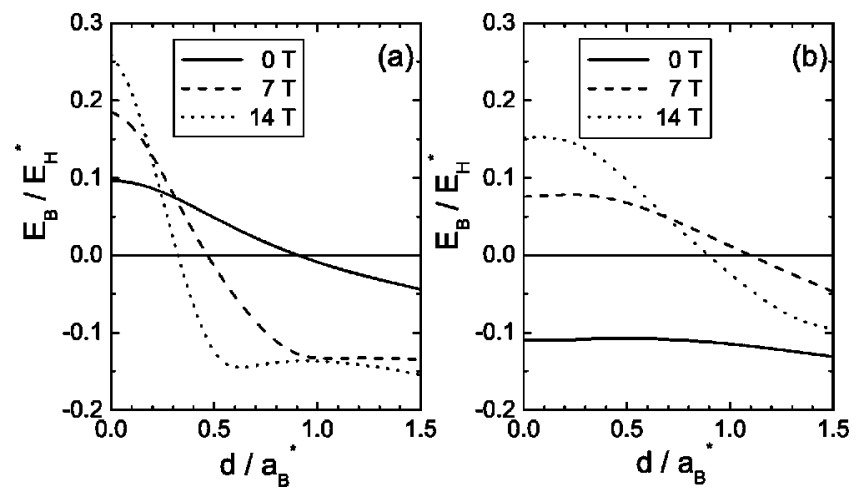

FIG. 4. The binding energies of ${ }^{1} S$ (a) and ${ }^{3} P$ (b) states as a function of interdot distance for three different values of magnetic field.

electron. In Fig. 4 we plot the binding energies of the lowest ${ }^{1} S$ and ${ }^{3} P$ states versus the interdot separation. We observe that binding persists only up to interdot distances around $a_{B}^{*}$ which is considerably less than the radii of the quantum dots $\left(2.5 a_{B}^{*}\right)$. Nevertheless, in our case the sensitivity of the trion binding energies on the interdot distance is not as dramatic as that predicted for asymmetric quantum wells. ${ }^{20}$

We see that with increasing magnetic field and interdot separation the angular momentum of the ground state follows a sequence of negative numbers growing in absolute value (given in parentheses in Fig. 3). The angular momentum switchings are accompanied by singlet-triplet transitions; thus all ground states of even angular momentum are spin singlets, while odd angular momenta correspond to triplet states. This kind of behavior is typical of interacting twoelectron systems; thus we may conclude that in our system the angular momentum transitions are induced by the strong Coulomb repulsion between the two electrons. The role of the hole is to screen the interelectron interaction. Thus, as the quantum dots are brought closer to each other, the angular momentum transitions are shifted towards higher values of the magnetic field or are even quenched. We find no transitions at $d=0$ for magnetic fields up to $30 \mathrm{~T}$.

One also observes that the area corresponding to the singlet ${ }^{1} D$ state in Fig. 3 is very narrow and is terminated at a magnetic field of about $9 \mathrm{~T}$. Furthermore, the following expected singlet state ${ }^{1} G$ is missing altogether. This effect is brought about by the inclusion of the Zeeman energy which linearly lowers the energy of the lowest component of the triplet states with increasing magnetic field. This is illustrated by Fig. 5 where we show the $B-d$ phase diagram in the absence of the Zeeman energy (i.e., $g_{e}^{*}=0$ ).

\section{B. Oscillator strengths}

Although in our model the electrons and the hole are treated as strictly two dimensional and moving in spatially separated layers, we can, nevertheless, obtain estimates for the photoluminescence oscillator strengths. To this end, we assume that the total wave function of the electron-hole system is a product of the computed in-plane part and an un-

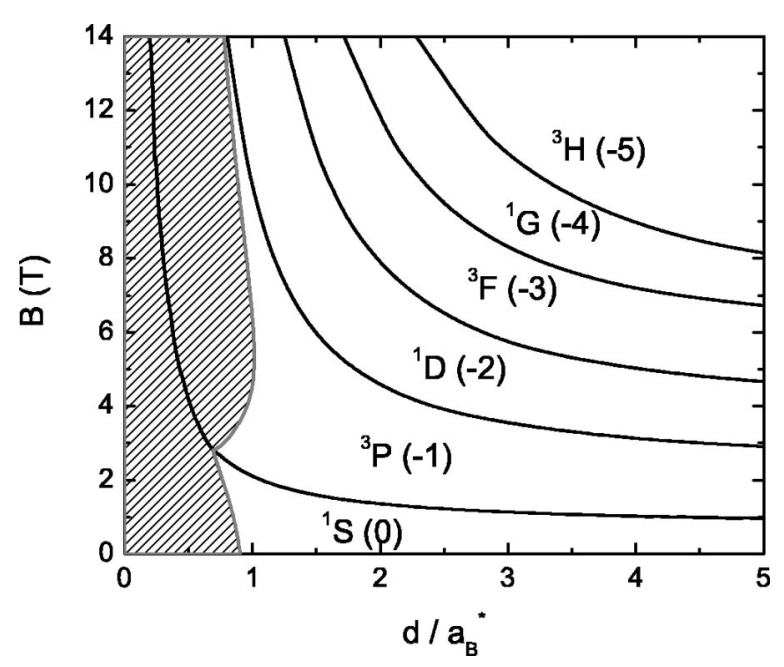

FIG. 5. The same as in Fig. 3 but now in the absence of the Zeeman interaction: i.e., $g_{e}^{*}=0$. In contrast to Fig. 3 we find the singlet ground state with angular momentum -4 . Also, the area belonging to the $\operatorname{state}^{1} D$ is no longer terminated at high magnetic fields.

known function of the perpendicular coordinate which describes the penetration of the particles into the barrier region between the dots. Then the overlap between the electron and hole wave functions will be expressed as an integral over the lateral coordinates times an unknown factor which is the same for all states. This allows us to compute the relative oscillator strengths of various transitions.

The lowest-energy component of the trion triplet state with $S_{e}^{z}+S_{h}^{z}=5 / 2$ is optically inactive ${ }^{30}$ since no transition can take it to a final state of a single electron with $S_{e}^{z}$ $= \pm 1 / 2$, thus lowering the total spin projection by at least 2 . Therefore, we will consider the transitions from the middle component of triplet states as well as transitions from singlet states. In both cases, the total spin projection in the initial state is $S_{e}^{z}+S_{h}^{z}=3 / 2$-i.e., one electron is in the spin-up state while the other is polarized spin down - and in the final state, we always have a single electron with $S_{e}^{z}=1 / 2$.

In our further treatment of the calculation of the oscillator strengths we follow Ref. 31 and write the initial and final states as

$$
\begin{gathered}
\left|\Phi_{i}\right\rangle=\sum_{\lambda_{1} \lambda_{2} \lambda_{h}} C_{\lambda_{1} \lambda_{2} \lambda_{h}} a_{\lambda_{1} \uparrow}^{\dagger} a_{\lambda_{2} \downarrow}^{\dagger} d_{\lambda_{h} \uparrow}^{\dagger}|0\rangle, \\
\left|\Phi_{f}\right\rangle=a_{\lambda \uparrow}^{\dagger}|0\rangle,
\end{gathered}
$$

where $a_{\lambda_{i}}^{\dagger}$ and $d_{\lambda_{i}}^{\dagger}$ are, respectively, the electron and hole creation operators in state $\left|\lambda_{i}\right\rangle$, the arrows $\uparrow, \downarrow$ denote spin polarizations, and the expansion coefficients $C_{\lambda_{1} \lambda_{2} \lambda_{h}}$ are obtained from the diagonalization of the Hamiltonian. For the sake of brevity we omit the specification of photon states. Since in the present calculation we assume the two quantum dots to have equal sizes, the lateral overlap of the electron and hole wave functions, 


$$
\int d^{2} r \psi_{e}\left(\lambda_{e} \mid \mathbf{r}\right) \psi_{h}\left(\lambda_{h} \mid \mathbf{r}\right),
$$

equals unity when the electron and hole are in "complementary" states $\lambda$ and $\bar{\lambda}$ with the same radial quantum number and angular quantum number differing in sign and is zero otherwise. Thus the interaction Hamiltonian describing optical emission attains the form

$$
H_{\text {int }} \propto \sum_{\lambda} a_{\lambda \downarrow} d_{\lambda \uparrow} b^{\dagger}
$$

hereby describing the annihilation of an electron and a hole in "complementary" states and creating a photon in a corresponding state. A straightforward calculation leads to the following expression for the oscillator strength of the transition to the final state with an electron in a given orbital $\lambda_{0}$ :

$$
f=\left|\sum_{\lambda} C_{\lambda_{0} \lambda \bar{\lambda}}\right|^{2} .
$$

The initial trion state is usually a superposition of many three-particle configurations. Therefore, after annihilating one electron with a hole the remaining electron may be found in any of a number of different final states with finite probabilities. However, since the two annihilating particles have zero total angular momentum, the angular momentum of eligible final states must equal the angular momentum of the trion. Moreover, in all cases considered below after the transition the remaining electron ends up in its lowest possible state with much higher probability than in any other state. Therefore, we compute the oscillator strengths only for this dominant transition.

In Fig. 6, we plot the oscillator strengths (in arbitrary units) for the optical transitions from the four lowest-energy states considered in the previous subsection as a function of magnetic field for two interdot distances $d=0.25$ and $d$ =1.0. One observes that the transitions from the trion $S$ states are as a rule stronger than those from the $P$ states. Since the electron-electron and electron-hole interactions are quite strong, in most cases there are many terms contributing to the sum in Eq. (12) and thus it is not always easy to interpret the strengths of different transitions microscopically by identifying a dominant initial-state configuration that sets the transition rate. However, in certain cases such an analysis becomes manageable.

For example, one may infer that the state ${ }^{1} S$ is always rather bright because in this state the most important singleparticle configuration is the one having both electrons and the hole in the lowest states with angular momentum 0 . Here and further in the text we will use the compact notation $(0,0 ; 0)$ listing the angular momenta of electrons first and then, separated by a semicolon, the one of the hole. It is easy to see that in such a state there is a large overlap between electron and hole wave functions. On the other hand, the trion state ${ }^{1} P$ is very dark at low magnetic fields. This fact can be traced back to the predominant configuration $(0,0$; -1 ) where the hole (due to the closer energy level spacing of the holes) is promoted to a higher orbital with angular
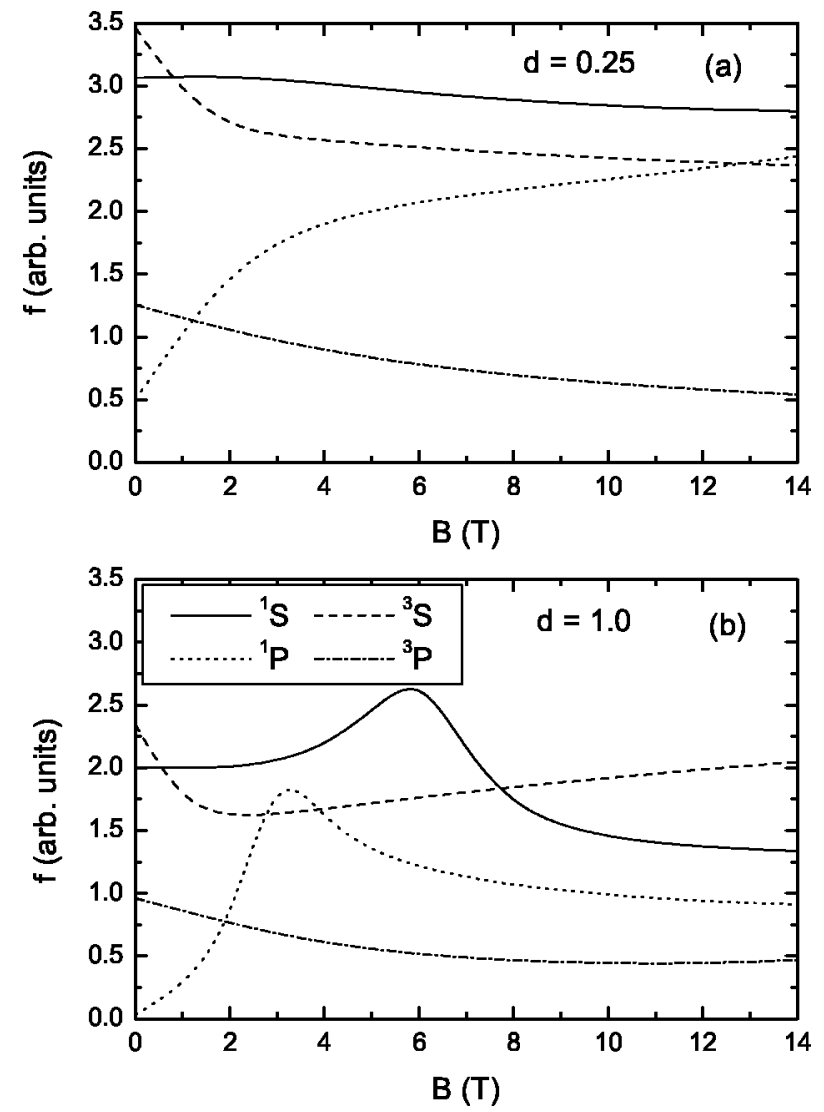

FIG. 6. The oscillator strengths for optical transitions from the four lowest trion energy levels at $d=0.25$ (a) and $d=1.0$ (b). The lower-energy triplet ${ }^{3} P$ is consistently darker than the higherenergy ${ }^{3} S$.

momentum -1 so that there is no overlap with the electrons in their ground state. With increasing magnetic field the energies of single-particle states are altered and this configuration is replaced by $(0,-1 ; 0)$, and consequently, the oscillator strength starts growing rapidly.

Taking a look at the triplet states we observe that the ${ }^{3} P$ state has a rather low oscillator strength and thus may be called a "dark" triplet state. This state is always lower in energy than the "bright" triplet state ${ }^{3} S$; i.e., the present situation resembles that encountered in the investigation of charged excitons in a two-dimensional electron gas. ${ }^{19}$

\section{Charge distribution in the dots}

As a next step, we look at the electron and hole charge distributions in the dots and their redistribution during the ground-state angular momentum transitions. Such charge redistributions result in abrupt changes of the spatial extent of the electron and hole subsystems and consequently in its capacitance. Recent vertical transport experiments performed on single quantum dots ${ }^{32}$ were able to detect such area changes of about $10 \%$.

We define the effective radius of the electron (hole) charge distribution by taking the square root of the expectation value of its squared radial coordinate: i.e., $R_{e(h)}$ $=\left\langle r_{e(h)}^{2}\right\rangle^{1 / 2}$. The results obtained in this way do not differ in 


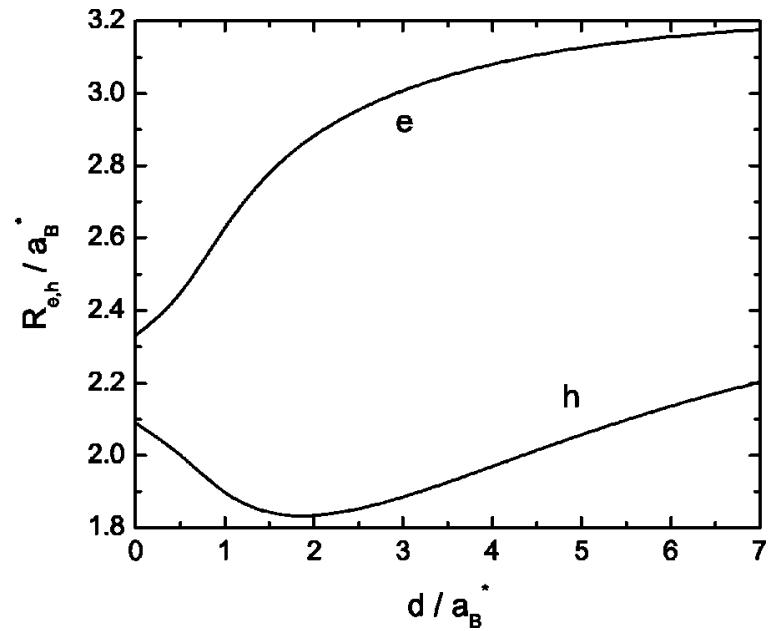

FIG. 7. The radii of the distribution of electrons (upper curve) and holes (lower curve) as a function of interdot distance $d$ at zero magnetic field. Note that the hole radius attains a minimum at a finite interdot distance.

essence from those obtained using an alternative definition for the radius ${ }^{33}$ formulated as a requirement that the circle of this radius should contain $95 \%$ of the total charge.

In Fig. 7, we show the dependences of the radius of the electron, $R_{e}$, and hole, $R_{h}$, distribution in the ground state $\left({ }^{1} S\right)$ as a function of interdot distance $d$ at $B=0 \mathrm{~T}$. Naturally, one observes that the radius of the electron distribution is decreasing monotonically when the two dots are brought closer to each other. This is easily explained by the fact that a nearby hole creates an additional centrally attractive potential in the dot with electrons, thereby compressing their radial distribution. The behavior of the hole dot is quite different. At large distances $d$ the dot is contracting as expected, however, when the interdot separation becomes smaller than approximately $1.8 a_{B}^{*}$ (this number is just slightly less than the confinement radii in the dots: i.e., $2.5 a_{B}^{*}$ ) the radius of the hole dot starts growing again. The hole feels the attractive potential of the electronic dot which is larger in size and, therefore, at close distances creates a net force directed towards the edge rather than the center.

With this result in mind, let us proceed to the magnetic field dependence of the charge distribution radii. The two panels of Fig. 8 show, respectively, the evolution of $R_{e}$ and $R_{h}$ versus the magnetic field at three fixed values of interdot distance $d=0.4,1.0$, and 2.0. We see that the electron radius increases with increasing interdot distance $d$. At the magnetic field values corresponding to angular momentum transitions (see Fig. 3) these curves exhibit quite substantial abrupt jumps on the order of $10 \%$ of their value, these jumps being comparable in magnitude for both closely spaced and more distant dots.

The behavior of the hole subsystem, shown in Fig. 8(b), is quite different. The curves appear in the same order as those pertaining to electrons at high magnetic fields, but we find the opposite arrangement at the lower end of the $B$ value range, and they intersect in the middle. The abrupt jumps are also notable but much smaller in magnitude if compared to the ones in Fig. 8(a). The radius of the hole distribution
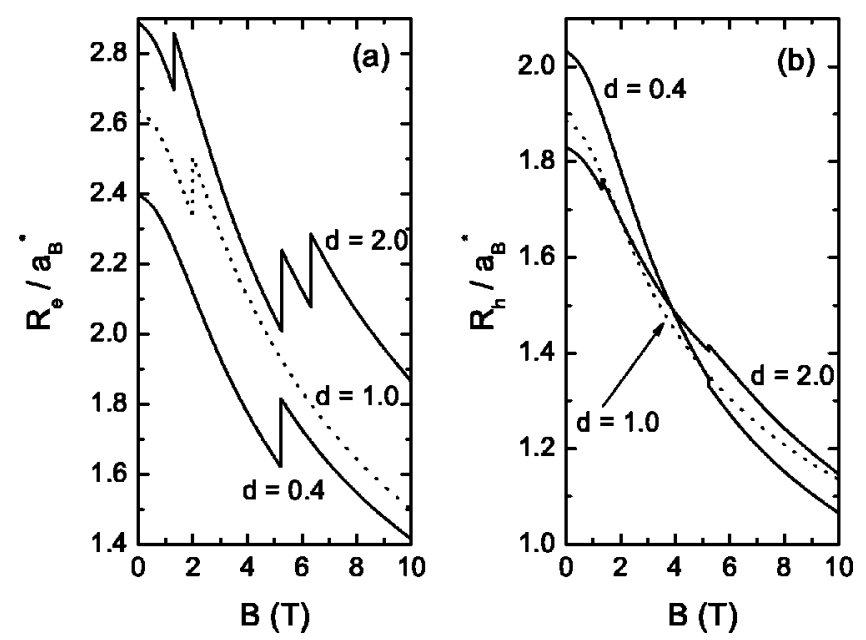

FIG. 8. The electron (a) and hole (b) distribution radii as a function of the magnetic field strength for three values of the interdot distance $d$. The abrupt jumps accompanying angular momentum transitions are apparent and are considerably stronger in the electronic subsystem.

typically changes by no more than $1 \%$ or $2 \%$, and moreover, this change may take place in either direction; i.e., the size of the hole dot may increase as well as decrease at the transition to a higher total angular momentum state.

\section{Correlation between the particles}

The angular momentum transitions also influence the relative arrangement of electrons and hole with respect to each other. In Fig. 9 we plot the average electron-electron and electron-hole separations defined as the square root of the ground-state expectation value of the radial distance between the two respective particles-i.e., $\rho_{e e}=\left\langle\left(\mathbf{r}_{e 1}\right.\right.$ $\left.\left.-\mathbf{r}_{e 2}\right)^{2}\right\rangle^{1 / 2}$ and $\rho_{e h}=\left\langle\left(\mathbf{r}_{e 1}-\mathbf{r}_{h}\right)^{2}\right\rangle^{1 / 2}$. We see that the general trend of both the electron-electron and electron-hole separation is very similar. Both distances $\rho_{e e}$ and $\rho_{e h}$ tend to de-
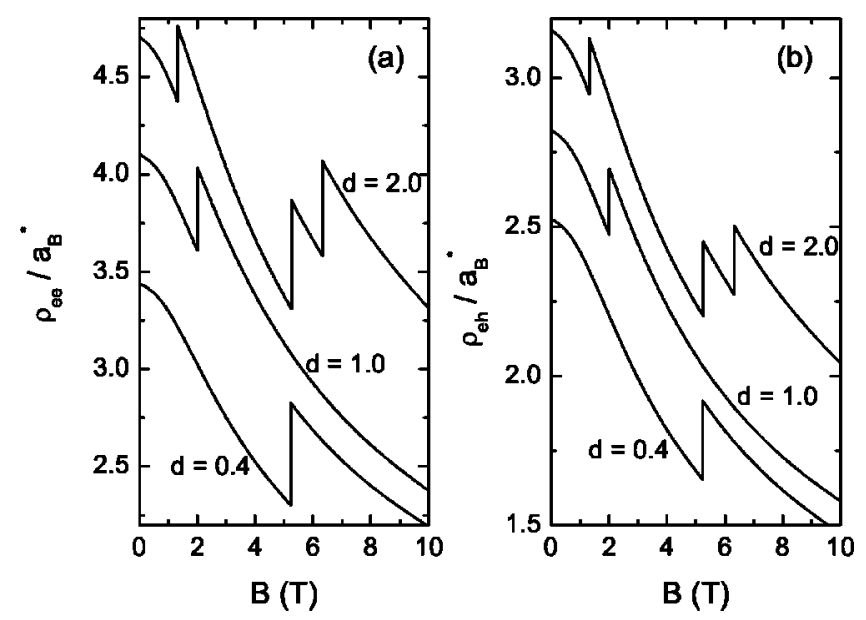

FIG. 9. The average electron-electron (a) and electron-hole (b) separation in the ground state. As in Fig. 8, abrupt increases in the interparticle distances at the angular momentum transitions are clearly visible. 


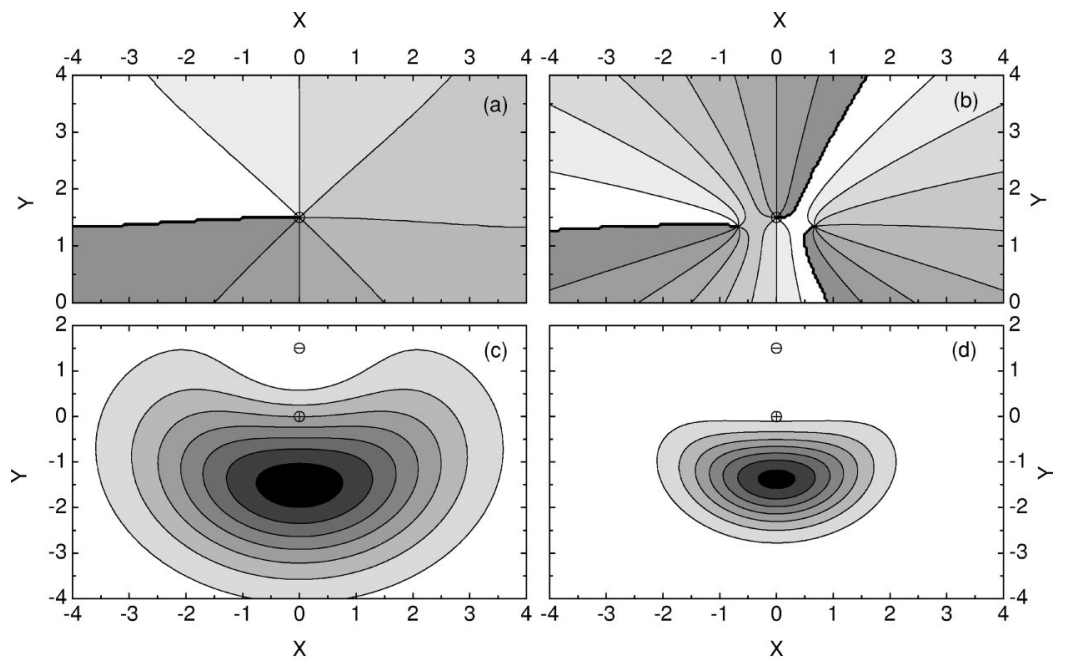

FIG. 10. The phase $[(a)$ and (b)] and the squared absolute value [(c) and (d)] of the reduced wave function of the trion calculated for fixed position of one of the electrons (at $x=0$, $y=1.5$ ) and hole (at $x=y=0$ ). The interdot separation is set to $d=1.5$. Panels (a) and (c) correspond to the magnetic field $B=2 \mathrm{~T}$ and ground-state angular momentum -1 , and panels (b) and (d) are drawn for $B=10 \mathrm{~T}$ and angular momentum -3 . The norm of the reduced wave function signifies the conditional probability to find the second electron. One and three vortices are seen in the panels (a) and (b) at and close to the position of the fixed electron. crease with growing magnetic field due to its localizing effect. This monotonous decrease is interrupted by abrupt jumps upwards in both curves at the angular momentum transition points.

In order to obtain a more detailed picture we also calculate the reduced electron wave function in the dot by evaluating the total three-particle wave function $\Psi\left(\mathbf{r}_{1}, \mathbf{r}_{2}, \mathbf{r}_{h}\right)$, at fixed positions of one electron and the hole. Figure 10 shows the result obtained for interdot distance $d=1.5$ and two magnetic field values of $B=2 \mathrm{~T}$ in the two left panels (a) and (c) and $B=10 \mathrm{~T}$ in the right panels (b) and (d). The hole is fixed at the center of the quantum $\operatorname{dot}(x=y=0)$ and marked by an encircled "+" sign while one of the electrons is fixed at a point with coordinates $x=0$ and $y=1.5$ and indicated by an encircled "- " sign.

In the panels (c) and (d) we plot the squared absolute value of the reduced ground-state wave function which describes the conditional probability to find the remaining electron at a given position. One observes that in both cases of high and low magnetic field this probability is highest on the opposite side of the hole from the fixed electron. The electron tends to be more strongly localized at higher magnetic fields. The upper panels (a) and (b) show the behavior of the phase of the reduced wave function close to the position of the fixed electron. Notice that points are found where at which the conditional probability is zero-e.g., at the position of the fixed electron - and where there is a circulation of the phase by $2 \pi$. Such a point is called a vortex. At low magnetic fields [panel (a)] we observe a single vortex, indicating that the wave function has a simple zero at this position. The ground-state angular momentum in this case is -1 . At higher magnetic fields we observe three vortices. One is attached to the position of the fixed electron while the other two are approaching it from the sides. Because of the presence of the hole, the latter two vortices are not positioned diagonally opposite with respect to the position of the electron. The appearance of three zeros of the reduced wave function indicates a much lower probability to find an electron in the vicinity of the fixed one. In this case, the ground state has angular momentum -3 .

\section{E. Dependence on the confining radius}

Up to now we considered coupled quantum dots in which the electrons and hole have lateral confinement lengths $l_{0}$ $=L_{0}=2.5 a_{B}^{*}$. It is also interesting to look at the dependence of the trion binding energy on the confinement strength in the dots. In Fig. 11 we plot the binding energies at zero magnetic field and various fixed interdot distances between $d=0$ and $d=1.25 a_{B}^{*}$ as a function of the confining lengths $l_{0}=L_{0}$. All curves, except for the one corresponding to $d=0$, tend to decrease rapidly towards large negative values (that is, the trion is unbound) at small values of the confinement length. (The dashed line corresponding to $d=0.25 a_{B}^{*}$ also follows the suit at very short confinement lengths.) This behavior can be understood by realizing that the strong in-plane confinement results in an increase of the effective interdot separation-i.e., its ratio to the lateral extent of the wave function. On the other hand, for the case of closely spaced dots (i.e., $d<0.25 a_{B}^{*}$ ) the strong lateral confinement (with the values of $l_{0}$ and $L_{0}$ close to $a_{B}^{*}$ ) allows us to obtain more tightly bound trions. Similar results were recently obtained for three-dimensional (finite thickness) quantum dots. ${ }^{25}$

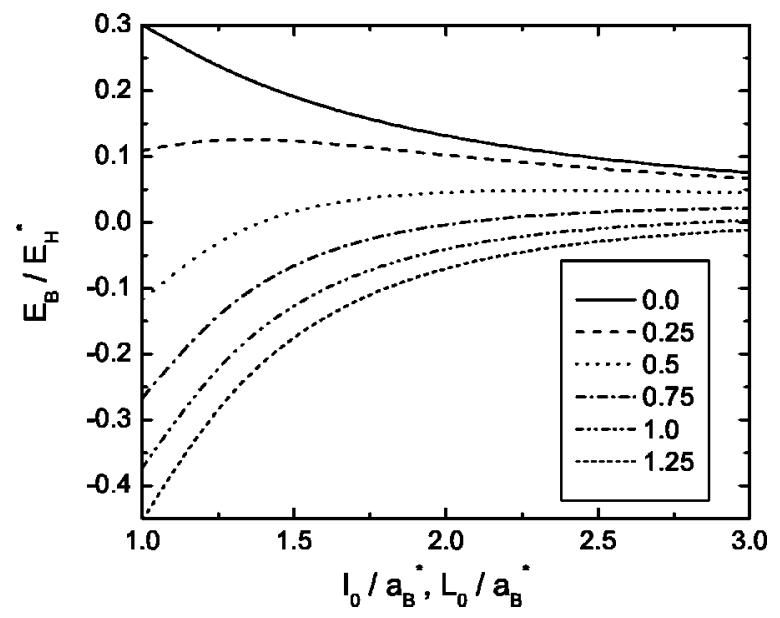

FIG. 11. The trion binding energy at zero magnetic field vs the confinement lengths $l_{0}=L_{0}$. Different curves correspond to different interdot distances. 


\section{SUMMARY}

In conclusion we performed a study of interacting complexes of two electrons and one hole confined in coupled quantum dots. We obtained the $B-d$ (i.e., magnetic field versus interdot distance) phase diagram of the system and identified angular momentum and spin multiplicity transitions with increasing magnetic field and/or increasing separation between the two dots. Such transitions are accompanied by abrupt increases in the radius of the electron charge distribution and the electron-electron and electron-hole separation. The radius of the hole distribution is, on the other hand, quite inert. At small distances between the dots the trions are bound while they become unbound for $d \gtrsim a_{B}^{*}$. The triplet state ${ }^{3} P$ has a substantially lower oscillator strength than the ${ }^{1} S$ and ${ }^{3} S$ states.

\section{ACKNOWLEDGMENTS}

This work was supported by the European Commission GROWTH program NANOMAT project under Contract No. GSRD-CT-2001-00545, the Belgian Interuniversity Attraction Poles (IUAP), and the Flemish Concerted Action (GOA) programs. E.A. was supported by EU under Contract No. HPMF-CT-2001-01195. The authors would like to thank Dr. A. V. Vagov and Dr. M. Bonitz for useful discussions.

\section{APPENDIX: COULOMB MATRIX ELEMENT}

We briefly discuss the evaluation of electron-hole Coulomb matrix elements

$$
\begin{aligned}
\langle 12|V| 34\rangle= & \int d^{2} r_{1} \int d^{2} r_{2} \psi_{e}^{*}\left(n_{1} m_{1} \mid \mathbf{r}_{1}\right) \psi_{h}^{*}\left(n_{2} m_{2} \mid \mathbf{r}_{2}\right) \\
& \times \frac{1}{\left|\mathbf{r}_{1}-\mathbf{r}_{2}+\mathbf{d}\right|} \psi_{e}\left(n_{3} m_{3} \mid \mathbf{r}_{1}\right) \psi_{h}\left(n_{4} m_{4} \mid \mathbf{r}_{2}\right)
\end{aligned}
$$

between the states defined in Eq. (5). To tackle Eq. (A1) we generalize the result derived previously ${ }^{34}$ for a singlecomponent system moving in a single layer. In the case of equal lateral extensions of the electron and hole wave functions $(l=L)$ we may closely follow the procedure of Ref. 34 based on switching to the midpoint and relative coordinates according to $\mathbf{r}_{1(2)}=\mathbf{R} \pm \vec{\rho} / 2$. All the resulting integrals are calculable analytically except for the radial integral over $\rho$ which reduces to a numerical evaluation of the function

$$
\mathcal{F}_{\alpha}(x)=\frac{1}{\Gamma\left(\frac{\alpha+1}{2}\right)} \int_{0}^{\infty} d t e^{-t} t^{\alpha / 2-1 / 2} \sqrt{\frac{t}{t+x}}
$$

normalized so that $\mathcal{F}_{\alpha}(0) \equiv 1$ which is obtained in the limit $d \rightarrow 0$. The final result reads

$$
\begin{aligned}
& \langle 12|V| 34\rangle=\delta_{m_{1}+m_{2}, m_{3}+m_{4}}\left[\prod_{i=1}^{4} \frac{n_{i} !}{\left(n_{i}+\left|m_{i}\right|\right) !}\right]^{1 / 2} \\
& \times \sum_{(4) j=0}^{n} \frac{(-1)^{j_{1}+j_{2}+j_{3}+j_{4}}}{j_{1} ! j_{2} ! j_{3} ! j_{4} !}\left[\prod_{i=1}^{4}\left(\begin{array}{l}
n_{i}+\left|m_{i}\right| \\
o p n_{i}-j_{i}
\end{array}\right)\right] \\
& \times \sum_{(4) s=0}^{\gamma} \delta_{s_{1}+s_{2}, s_{3}+s_{4}}(-1)^{\gamma_{2}+\gamma_{4}-s_{2}-s_{4}} \\
& \times\left[\prod_{i=1}^{4}\left(\begin{array}{c}
\gamma_{i} \\
s_{i}
\end{array}\right)\right] \Gamma\left(\frac{S+2}{2}\right) \Gamma\left(\frac{G-S+1}{2}\right) \mathcal{F}_{G-S}\left(\frac{d^{2}}{2 l^{2}}\right),
\end{aligned}
$$

with

$$
\begin{gathered}
G=\sum_{i=1}^{4} \gamma_{i}, S=\sum_{i=1}^{4} s_{i}, \sum_{(4) j=0}^{n} \equiv \sum_{j_{1}=0}^{n_{1}} \sum_{j_{2}=0}^{n_{2}} \sum_{j_{3}=0}^{n_{3}} \sum_{j_{4}=0}^{n_{4}}, \\
\gamma_{1}=\left(\left|m_{1}\right|+m_{1}\right) / 2+\left(\left|m_{3}\right|-m_{3}\right) / 2+j_{1}+j_{3}, \\
\gamma_{2}=\left(\left|m_{2}\right|+m_{2}\right) / 2+\left(\left|m_{4}\right|-m_{4}\right) / 2+j_{2}+j_{4}, \\
\gamma_{3}=\left(\left|m_{1}\right|-m_{1}\right) / 2+\left(\left|m_{3}\right|+m_{3}\right) / 2+j_{1}+j_{3}, \\
\gamma_{4}=\left(\left|m_{2}\right|-m_{2}\right) / 2+\left(\left|m_{4}\right|+m_{4}\right) / 2+j_{2}+j_{4} .
\end{gathered}
$$

If the electron and hole have different lateral extensions of the wave function, the above approach is still usable; however, one has to introduce "weighted" midpoint and relative coordinates according to

$$
\mathbf{r}_{1}=\mathbf{R}+q \vec{\rho}, \quad \mathbf{r}_{2}=\mathbf{R}-p \vec{\rho},
$$

with $p=L^{2} /\left(l^{2}+L^{2}\right)$ and $q=l^{2} /\left(l^{2}+L^{2}\right)$. In the case of equal confinement frequencies in the two dots and no magnetic field the weighting factors $p$ and $q$ are proportional to the masses of the respective particles.
${ }^{1}$ M.A. Lampert, Phys. Rev. Lett. 1, 450 (1958).

${ }^{2}$ T. Kawabata, K. Muro, and S. Narita, Solid State Commun. 23, 267 (1977).

${ }^{3}$ G.A. Thomas and T.M. Rice, Solid State Commun. 23, 359 (1977).

${ }^{4}$ B. Stébé, T. Sauder, M. Certier, and C. Comte, Solid State Commun. 26, 637 (1978).

${ }^{5}$ T. Ishii, M. Taniguchi, A. Kakizaki, K. Naito, H. Sugawara, and I. Nagakura, Phys. Rev. B 33, 5664 (1986).

${ }^{6}$ B. Stébé and A. Ainane, Superlattices Microstruct. 5, 545 (1989).
${ }^{7}$ B. Stébé, G. Munschy, L. Stauffer, F. Dujardin, and J. Murat, Phys. Rev. B 56, 12454 (1997).

${ }^{8}$ K. Kheng, R.T. Cox, M.Y. d'Aubigne, F. Bassani, K. Saminadayar, and S. Tatarenko, Phys. Rev. Lett. 71, 1752 (1993).

${ }^{9}$ G. Finkelstein, H. Shtrikman, and I. Bar-Joseph, Phys. Rev. B 53, 12593 (1996).

${ }^{10}$ A.J. Shields, J.L. Osborne, D.M. Whittaker, M.Y. Simmons, M. Pepper, and D.A. Ritchie, Phys. Rev. B 55, 1318 (1997).

${ }^{11}$ M. Hayne, C.L. Jones, R. Bogaerts, C. Riva, A. Usher, F.M. Peeters, F. Herlach, V.V. Moshchalkov, and M. Henini, Phys. 
Rev. B 59, 2927 (1999).

${ }^{12}$ R.J. Warburton, C.S. Dürr, K. Karrai, J.P. Kotthaus, G. MedeirosRibeiro, and P.M. Petroff, Phys. Rev. Lett. 79, 5282 (1997).

${ }^{13}$ V.I. Yudson, Phys. Rev. Lett. 77, 1564 (1996).

${ }^{14}$ A.O. Govorov and A.V. Chaplik, JETP Lett. 66, 454 (1997).

${ }^{15}$ E.S. Moskalenko, K.F. Karlsson, P.O. Holtz, B. Monemar, W.V. Schoenfeld, J.M. Garcia, and P.M. Petroff, J. Appl. Phys. 92, 6787 (2002).

${ }^{16}$ J.-Y. Marzin, J.-M. Gerard, A. Izraël, D. Barrier, and G. Bastard, Phys. Rev. Lett. 73, 716 (1994).

${ }^{17}$ A. Hartmann, Y. Ducommun, E. Kapon, U. Hohenester, and E. Molinari, Phys. Rev. Lett. 84, 5648 (2000).

${ }^{18}$ F.M. Peeters, C. Riva, and K. Varga, Physica B 300, 139 (2001).

${ }^{19}$ A. Wójs, J.J. Quinn, and P. Hawrylak, Phys. Rev. B 62, 4630 (2000)

${ }^{20}$ I. Szlufarska, A. Wójs, and J.J. Quinn, Phys. Rev. B 63, 085305 (2001).

${ }^{21}$ J.J. Palacios, D. Yoshioka, and A.H. MacDonald, Phys. Rev. B 54, 2296 (1996).

${ }^{22}$ A.B. Dzyubenko and A.Yu. Sivachenko, Phys. Rev. Lett. 84, 4429 (2000).

${ }^{23}$ T. Vanhoucke, M. Hayne, M. Henini, and V.V. Moshchalkov,
Phys. Rev. B 65, 233305 (2002).

${ }^{24}$ B. Szafran, B. Stébé, J. Adamowski, and S. Bednarek, J. Phys.: Condens. Matter 12, 2453 (2000).

${ }^{25}$ B. Szafran, B. Stébé, J. Adamowski, and S. Bednarek, Phys. Rev. B 66, 165331 (2002).

${ }^{26}$ A. Wójs and P. Hawrylak, Phys. Rev. B 51, 10880 (1995).

${ }^{27}$ W.D. Sheng and J.-P. Leburton, Appl. Phys. Lett. 81, 4449 (2002).

${ }^{28}$ E. Anisimovas and F.M. Peeters, Phys. Rev. B 65, 233302 (2002).

${ }^{29}$ A.V. Filinov, M. Bonitz, and Yu.E. Lozovik, J. Phys. A 36, 5899 (2003).

${ }^{30}$ T. Vanhoucke, M. Hayne, M. Henini, and V.V. Moshchalkov, Phys. Rev. B 63, 125331 (2001).

${ }^{31}$ B. Stébé, E. Feddi, A. Ainane, and F. Dujardin, Phys. Rev. B 58, 9926 (1998).

${ }^{32}$ T.H. Oosterkamp, J.W. Janssen, L.P. Kouwenhoven, D.G. Austing, T. Honda, and S. Tarucha, Phys. Rev. Lett. 82, 2931 (1999).

${ }^{33}$ P.A. Maksym, H. Imamura, G.P. Mallon, and H. Aoki, J. Phys.: Condens. Matter 12, 299 (2000).

${ }^{34}$ E. Anisimovas and A. Matulis, J. Phys.: Condens. Matter 10, 601 (1998). 